\title{
Micromorphic vs. Phase-Field Approaches for Gradient Viscoplasticity and Phase Transformations
}

\author{
Samuel Forest, Kais Ammar, and Benoît Appolaire
}

\begin{abstract}
Strain gradient models and generalized continua are increasingly used to introduce characteristic lengths in the mechanical behavior of materials with microstructure. On the other hand, phase-field models have proved to be efficient tools to simulate microstructure evolution due to thermodynamical processes in the presence of mechanical deformation. It is shown that both methods have strong connections from the point of view of thermomechanical field theory. A general formulation of thermomechanics with additional degrees of freedom is presented that encompasses both applications as special cases. It is based on the introduction of additional power of internal forces introducing generalized stresses. The current knowledge in the formulation of physically non-linear constitutive equations is used to develop strongly coupled elastoviscoplastic material models involving phase transformation and moving boundaries.
\end{abstract}

\section{Introduction}

There are strong links between generalized continuum mechanics and phase field models which are striving in modern field theories of materials. Mindlin's and Casal's second gradient model of mechanics and the Cahn-Hilliard diffusion theory were developed almost simultaneously. More generally, the necessity of introducing additional degrees of freedom in continuum models arose in the 1960s in order to account for microstructure effects on the overall material's response. However, generalized continuum mechanics, with paradigms like Eringen's micromorphic model

Samuel Forest · Kais Ammar

Mines ParisTech, Centre des Matériaux, CNRS UMR 7633, BP 87,

91003 Evry Cedex, France

e-mail: \{samuel. forest, kais . ammar\}@ensmp.fr

Benoît Appolaire

LEM, ONERA/CNRS, 29 Avenue de la Division Leclerc, BP 72, 92322 Châtillon, France e-mail: benoit. appolaire@onera.fr

B. Markert (Ed.): Advances in Extended \& Multifield Theories for Continua, LNACM 59, pp. 69-88 springerlink.com

(C) Springer-Verlag Berlin Heidelberg 2011 
and Aifantis strain gradient plasticity, developed along an independent track from phase-field approach embodied by Khachaturyan's views, for instance.

The links have been seen recently within the context of plasticity and damage mechanics. The computational mechanics community aimed at introducing the evolution of microstructures into their simulations [2, 38] whereas physicists started introducing plasticity into the thermodynamical setting [19]. Cooperation between these communities becomes necessary when tackling damage mechanics and crack propagation simulation [6, 32]. First attempts to present a general constitutive framework encompassing classical enhanced mechanical and thermodynamical models have been proposed recently [7, 14, 30]. Such an approach is presented in this chapter and extended to sophisticated descriptions of interactions between viscoplasticity and phase transformations.

The micromorphic model originates from Eringen's introduction of microdeformation tensor at each material point that accounts for the changes of a triad of microstructure vectors. In the present chapter, the micromorphic approach denotes an extension of this theory to other variables than total deformation, namely plastic strain, hardening variables, and even temperature and concentration. The gist of the micromorphic model is to associate a microstructure quantity (e.g. microdeformation) to an overall quantity (e. g. macroscopic deformation). The deviation of the microvariable from the macrovariable and the gradient of the microvariable are sources of stored energy and dissipation. They are controlled by generalized stresses which contribute to the power of internal forces.

On the other hand, the phase-field approach has proved to be an efficient method to model the motion of interfaces and growth of precipitates based on a sound thermodynamical formulation including non convex free energy potentials [13]. The effect of microelasticity on the morphological aspects and kinetics of phase transformation is classically studied but the occurrence of plasticity is recent [19, 20, 38]. Beyond plasticity, damage and crack propagation are the subject of both generalized continuum and phase-field approaches [6, 17, 31, 32, 39]. Phase-field simulations usually rely on finite differences or fast Fourier methods. More recently, the finite element method was also used in order to tackle more general boundary conditions [2, 32, 36].

The objective of the present chapter is to formulate a thermomechanical theory of continua with additional degrees of freedom. It is shown in a first part that the theory encompasses available generalized continuum theories and phase-field models provided that well-suited free energy and dissipation potentials are selected. The current strain gradient plasticity models are then extended to account simultaneously for plastic strain gradient and plastic strain rate gradient in order to address viscoplastic instabilities occurring in metal plasticity like dynamic strain aging. The second part of the work exposes how the well-known elastoviscoplastic constitutive framework can be incorporated into the available phase-field approach in order to investigate the coupling between viscoplasticity and phase transformation. An original approach is proposed that resorts to standard homogenization techniques used in the mechanics of heterogeneous materials. 
Intrinsic notations are used throughout this work. In particular, scalars, vectors, tensors of second, third and fourth ranks are denoted by $a, \mathbf{a}, \stackrel{2}{\mathbf{a}}, \underset{\mathbf{a}}{\mathbf{a}}, \stackrel{4}{\mathbf{a}}$, respectively. Contractions are written as:

$$
\mathbf{a} \cdot \mathbf{b}=a_{i} b_{i}, \quad \stackrel{2}{\mathbf{a}}: \stackrel{2}{\mathbf{b}}=a_{i j} b_{i j}, \quad \stackrel{3}{\mathbf{a}}: \mathbf{3}=a_{i j k} b_{i j k}
$$

using the Einstein summation rule for repeated indices. The gradient operator $\boldsymbol{\nabla}$ is introduced as

$$
\mathbf{u} \otimes \boldsymbol{\nabla}=u_{i, j} \mathbf{e}_{i} \otimes \mathbf{e}_{j}, \quad \text { with } \quad u_{i, j}=\frac{\partial u_{i}}{\partial x_{j}},
$$

where $\left(\mathbf{e}_{i}\right)_{i=1,2,3}$ is a Cartesian orthonormal basis.

For the sake of brevity, the analysis is limited to the small deformation framework throughout this work. Also most situations are considered under isothermal conditions.

\section{Thermomechanics with Additional Degrees of Freedom}

\subsection{General Setting}

The displacement variables of mechanics can be complemented by additional degrees of freedom (DOF), $\phi$, that can be scalars as well as tensor variables of given rank:

$$
D O F=\{\mathbf{u}, \quad \phi\} .
$$

A first gradient theory is built on the basis of this set of degrees of freedom:

$$
S T R A I N=\{\stackrel{2}{\varepsilon}, \quad \phi, \quad \nabla \phi\} .
$$

The strain tensor, $\stackrel{2}{\varepsilon}$, is the symmetric part of the gradient of the displacement field. The main assumption of the proposed theory is that the gradient of the additional degrees of freedom contribute to the work of internal forces in the energy equation, in contrast to internal variables and concentration in diffusion theory. Depending on the invariance properties of the variable $\phi$, it can itself contribute to the work of internal forces together with its gradient. It is not the case for the displacement itself which is not an objective vector. The virtual power of internal forces is then extended to the virtual power done by the additional variable and its first gradient:

$$
\begin{gathered}
\mathcal{P}^{(i)}\left(\dot{\mathbf{u}}^{\star}, \dot{\phi}^{\star}\right)=-\int_{\mathcal{D}} p^{(i)}\left(\dot{\mathbf{u}}^{\star}, \dot{\phi}^{\star}\right) d V, \\
p^{(i)}\left(\dot{\mathbf{u}}^{\star}, \dot{\phi}^{\star}\right)=\stackrel{2}{\sigma}: \nabla \dot{\mathbf{u}}^{\star}+a \dot{\phi}^{\star}+\mathbf{b} \cdot \boldsymbol{\nabla} \dot{\phi}^{\star},
\end{gathered}
$$


where $\mathcal{D}$ is a subdomain of the current configuration $\Omega$ of the body. Stars denote virtual fields. The Cauchy stress tensor is $\stackrel{2}{\sigma}$ and $a$ and $\mathbf{b}$ are generalized stresses associated with the additional DOF and its first gradient, respectively. Similarly, the power of contact forces must be extended as follows:

$$
\mathcal{P}^{(c)}\left(\dot{\mathbf{u}}^{\star}, \dot{\phi}^{\star}\right)=\int_{\mathcal{D}} p^{(c)}\left(\dot{\mathbf{u}}^{\star}, \dot{\phi}^{\star}\right) d V, \quad p^{(c)}\left(\dot{\mathbf{u}}^{\star}, \dot{\phi}^{\star}\right)=\mathbf{t} \cdot \dot{\mathbf{u}}^{\star}+a^{c} \dot{\phi}^{\star}
$$

where $\mathbf{t}$ is the traction vector and $a^{c}$ a generalized traction. In general, the power of forces acting at a distance must also be extended in the form:

$$
\mathcal{P}^{(e)}\left(\dot{\mathbf{u}}^{\star}, \dot{\phi}^{\star}\right)=\int_{\mathcal{D}} p^{(e)}\left(\dot{\mathbf{u}}^{\star}, \dot{\phi}^{\star}\right) d V, \quad p^{(e)}\left(\dot{\mathbf{u}}^{\star}, \dot{\phi}^{\star}\right)=\rho \mathbf{f} \cdot \dot{\mathbf{u}}^{\star}+a^{e} \dot{\phi}^{\star}+\mathbf{b}^{e} \cdot \nabla \dot{\phi}^{\star}
$$

where $\rho \mathbf{f}$ accounts for given simple body forces and $a^{e}$ for generalized volume forces. The power of inertial forces also requires, for the sake of generality, the introduction of an inertia $I$ associated with the acceleration of the additional degrees of freedom:

$$
\mathcal{P}^{(a)}\left(\dot{\mathbf{u}}^{\star}, \dot{\phi}^{\star}\right)=\int_{\mathcal{D}} p^{(a)}\left(\dot{\mathbf{u}}^{\star}, \dot{\phi}^{\star}\right) d V, \quad p^{(a)}\left(\dot{\mathbf{u}}^{\star}, \dot{\phi}^{\star}\right)=-\rho \ddot{\mathbf{u}} \cdot \dot{\mathbf{u}}^{\star}-I \ddot{\phi} \dot{\phi}^{\star}
$$

Following [21], given body couples and double forces working with the gradient of the velocity field, could also be introduced in the theory. The generalized principle of virtual power with respect to the velocity and additional DOF, is formulated as

$$
\mathcal{P}^{(i)}\left(\dot{\mathbf{u}}^{\star}, \dot{\phi}^{\star}\right)+\mathcal{P}^{(e)}\left(\dot{\mathbf{u}}^{\star}, \dot{\phi}^{\star}\right)+\mathcal{P}^{(c)}\left(\dot{\mathbf{u}}^{\star}, \dot{\phi}^{\star}\right)+\mathcal{P}^{(a)}\left(\dot{\mathbf{u}}^{\star}, \dot{\phi}^{\star}\right)=0, \forall \mathcal{D} \subset \Omega, \forall \dot{\mathbf{u}}^{\star}, \dot{\phi}
$$

The method of virtual power according to [27] is used then to derive the standard local balance of momentum equation:

$$
\boldsymbol{\nabla} \cdot \stackrel{2}{\boldsymbol{\sigma}}+\rho \mathbf{f}=\rho \ddot{\mathbf{u}}, \quad \forall \mathbf{x} \in \Omega
$$

and the generalized balance of micromorphic momentum equation:

$$
\boldsymbol{\nabla} \cdot\left(\mathbf{b}-\mathbf{b}^{e}\right)-a+a^{e}=I \ddot{\phi}, \quad \forall \mathbf{x} \in \Omega .
$$

The method also delivers the associated boundary conditions for the simple and generalized tractions:

$$
\mathbf{t}=\stackrel{2}{\boldsymbol{\sigma}} \cdot \mathbf{n}, \quad a^{c}=\left(\mathbf{b}-\mathbf{b}^{e}\right) \cdot \mathbf{n}, \quad \forall \mathbf{x} \in \partial \mathcal{D}
$$

The local balance of energy is also enhanced by the generalized power already included in the power of internal forces (5):

$$
\rho \dot{\epsilon}=p^{(i)}-\boldsymbol{\nabla} \cdot \mathbf{q}+\rho r,
$$


where $\epsilon$ is the specific internal energy, $\mathbf{q}$ the heat flux vector and $r$ denotes external heat sources. The entropy principle takes the usual local form:

$$
-\rho(\dot{\psi}+\eta \dot{T})+p^{(i)}-\frac{\mathbf{q}}{T} \cdot \nabla T \geq 0,
$$

where it is assumed that the entropy production vector is still equal to the heat vector divided by temperature, as in classical thermomechanics. Again, the enhancement of the theory goes through the enriched power density of internal forces (5). The entropy principle is exploited according to classical continuum thermodynamics to derive the state laws. At this stage it is necessary to be more specific on the dependence of the state functions $\psi, \eta, \stackrel{2}{\sigma}, a, \mathbf{b}$ on state variables and to distinguish between dissipative and non-dissipative mechanisms. The introduction of dissipative mechanisms may require an increase in the number of state variables. These different situations are considered in the following subsections.

\subsection{Micromorphic Model as a Special Case}

The micromorphic model as initially proposed by Eringen [12] and Mindlin [33] amounts to introducing a generally non compatible microdeformation field:

$$
\phi \equiv \stackrel{2}{\chi},
$$

where ${ }_{\mathcal{X}}^{\mathcal{X}}$ is a generally non-symmetric second order tensor defined at each material point. When the microdeformation reduces to its skew symmetric part, the Cosserat model is retrieved [10, 16]. The microdeformation is to be compared to the deformation gradient:

$$
\stackrel{2}{\mathbf{e}}=\mathbf{u} \otimes \boldsymbol{\nabla}-\stackrel{2}{\chi}
$$

If the internal constraint $\stackrel{2}{\mathbf{e}}^{2} \equiv 0$ is enforced, the microdeformation coincides with the deformation and the micromorphic model reduces to Mindlin's second gradient theory. The free energy density depends of the following state variables:

$$
\text { STATE }=\{\stackrel{2}{\boldsymbol{\varepsilon}}, \quad \stackrel{2}{\mathbf{e}}, \quad \stackrel{3}{\mathbf{K}}:=\stackrel{2}{\chi} \otimes \nabla, \quad T, \quad \alpha\},
$$

where $\alpha$ denotes the set of internal variables required to represent dissipative mechanical phenomena. The Clausius-Duhem inequality (14) becomes, in the isothermal case,

$$
\left(\stackrel{2}{\boldsymbol{\sigma}}-\rho \frac{\partial \psi}{\partial \boldsymbol{\varepsilon}}\right): \stackrel{2}{\dot{\boldsymbol{\varepsilon}}}+\left(\stackrel{2}{\mathbf{a}}-\rho \frac{\partial \psi}{\partial \mathbf{e}}\right): \stackrel{2}{\dot{\mathbf{e}}}+\left(\stackrel{3}{\mathbf{b}}-\rho \frac{\partial \psi}{\partial \mathbf{K}}\right): \stackrel{3}{\dot{\mathbf{K}}}-\left(\rho \eta+\rho \frac{\partial \psi}{\partial T}\right) \dot{T}-\rho \frac{\partial \psi}{\partial T} \dot{\alpha} \geq 0,
$$


where $\mathbf{a}^{2}$ was taken as the stress conjugate to the relative deformation rate $\stackrel{2}{\dot{\mathbf{e}}}$ in the power of internal forces, which corresponds to an alternative form for [5]. The state laws for micromorphic media are obtained by assuming that the first four contribution are non-dissipative:

$$
\stackrel{2}{\boldsymbol{\sigma}}=\rho \frac{\partial \psi}{\partial \boldsymbol{\varepsilon}}, \quad 2=\rho \frac{\partial \psi}{\partial \mathbf{\varepsilon}}, \quad \stackrel{3}{\mathbf{b}}=\rho \frac{\partial \psi}{\partial \mathbf{K}}, \quad \eta=-\frac{\partial \psi}{\partial T} .
$$

Elastoviscoplastic micromorphic media are then obtained by a specific choice of the internal variables $\alpha$ and their evolution rules [16].

\subsection{Phase-Field Model as a Special Case}

Enhancing the mechanical power in the energy balance is plausible in the presence of microstructure induced mechanical phenomena, as proposed by Eringen. However, this is also possible in other contexts, namely when the DOF $\phi$ has a more general meaning of an order parameter. Fried and Gurtin [18, 22] suggested to consider the following reduced state space:

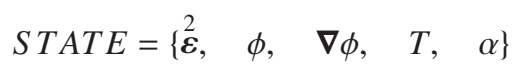

and the following state laws

$$
\stackrel{2}{\boldsymbol{\sigma}}=\rho \frac{\partial \psi}{\partial \boldsymbol{\varepsilon}}, \quad \mathbf{b}=\rho \frac{\partial \psi}{\partial \boldsymbol{\nabla} \phi}, \quad \eta=-\frac{\partial \psi}{\partial T},
$$

so that, in the isothermal case, the dissipation rate reduces to

$$
a^{v} \dot{\phi}+X \dot{\alpha} \geq 0 \quad \text { with } \quad a^{v}=a-\rho \frac{\partial \psi}{\partial \phi}, \quad X=-\rho \frac{\partial \psi}{\partial \alpha} .
$$

The choice of a convex potential $\Omega\left(a^{v}, X\right)$ providing the evolution laws

$$
\dot{\phi}=\frac{\partial \Omega}{\partial a^{v}}, \quad \dot{\alpha}=\frac{\partial \Omega}{\partial X}
$$

ensures the positivity of the dissipation rate.

As an illustration, let us consider a quadratic contribution of $a^{v}$ to the dissipation potential. We are led to the following relationships

$$
\dot{\phi}=\frac{1}{\beta} a^{v}=\frac{1}{\beta}\left(a-\rho \frac{\partial \psi}{\partial \phi}\right),
$$

where $\beta$ is a material parameter. The latter equation can be combined with the balance law (11), in the absence of volume or inertial forces, and the state law (19) to derive 


$$
\beta \dot{\phi}=\nabla \cdot\left(\rho \frac{\partial \psi}{\partial \nabla \phi}\right)-\rho \frac{\partial \psi}{\partial \phi},
$$

which corresponds to a general Ginzburg-Landau equation.

The authors in [38] have combined the micromorphic approach and the Cahn-Hilliard approach to diffusion in order to derive an alternative equation to Cahn-Hilliard.

\section{Constitutive Framework for Gradient and Micromorphic Viscoplasticity}

We now exploit the established general structure to propose a constitutive framework for elastoviscoplastic materials exhibiting plastic strain gradient. The attention is focused on an isotropic elastoviscoplastic medium characterized by the cumulated plastic strain, $p$. The proposed formulation encompasses Aifantis-like strain gradient plasticity models and introduces additional strain rate gradient effects. The total strain is split into its elastic and plastic parts: $\stackrel{2}{\varepsilon}=\stackrel{2}{\varepsilon}^{e}+\stackrel{2}{\boldsymbol{\varepsilon}}^{p}$. In this context, the additional DOF $\phi$ has the meaning of a microplastic strain [15] to be compared with $p$ itself.

Two variants of the constitutive framework are considered which handle in a slightly different way the dissipative contribution due to the generalized stresses.

\subsection{Introduction of Viscous Generalized Stresses}

The free energy density is assumed to depend on the following state variables:

$$
S T A T E=\left\{\stackrel{2}{\varepsilon}^{e}, \quad e:=\phi-p, \quad p, \quad \mathbf{K}:=\boldsymbol{\nabla} \phi\right\}
$$

The isothermal Clausius-Duhem inequality take the form:

$$
\left(\stackrel{2}{\boldsymbol{\sigma}}-\rho \frac{\partial \psi}{\partial \boldsymbol{\varepsilon}^{e}}\right): \stackrel{2}{\dot{\boldsymbol{\varepsilon}}}^{e}+\left(a-\rho \frac{\partial \psi}{\partial e}\right) \dot{e}+\left(\mathbf{b}-\rho \frac{\partial \psi}{\partial \mathbf{K}}\right) \cdot \dot{\mathbf{K}}+\stackrel{2}{\boldsymbol{\sigma}}: \stackrel{2}{\boldsymbol{\varepsilon}}^{p}+a \dot{p}-\rho \frac{\partial \psi}{\partial T} \dot{\alpha} \geq 0
$$

The following state laws are adopted:

$$
\stackrel{2}{\boldsymbol{\sigma}}=\rho \frac{\partial \psi}{\partial \dot{\boldsymbol{\varepsilon}}^{e}}, \quad R=\rho \frac{\partial \psi}{\partial p}
$$

To ensure the positivity of the dissipation rate associated with the generalized stress $a$ and $\mathbf{b}$, we adopt the viscoelastic constitutive equations

$$
a=\rho \frac{\partial \psi}{\partial e}+\beta \dot{e}, \quad \mathbf{b}=\rho \frac{\partial \psi}{\partial \mathbf{K}}+\kappa \dot{\mathbf{K}}
$$


where $\beta$ and $\kappa$ are generalized viscosity coefficients. This viscoelastic formulation amounts to splitting the generalized stresses $a$ and $\mathbf{b}$ into elastic (reversible) and viscous parts. Regarding viscoplastic deformation, a viscoplastic potential $\Omega(\stackrel{2}{\sigma}, a-$ $R$ ) is chosen such that

$$
\stackrel{2}{\dot{\boldsymbol{\varepsilon}}^{p}}=\frac{\partial \Omega}{\partial \dot{\boldsymbol{\sigma}}}, \quad \dot{p}=\frac{\partial \Omega}{\partial a-R} .
$$

In order to evidence the kind of gradient elastoviscoplastic models we aim at, we illustrate the case of a quadratic free energy potential:

$$
\begin{aligned}
\rho \psi & =\frac{1}{2} \boldsymbol{\varepsilon}^{e}: \stackrel{4}{\mathbf{C}}: \stackrel{2}{\boldsymbol{\varepsilon}}^{e}+R_{0} p+\frac{1}{2} H p^{2}+\frac{1}{2} H_{\phi} e^{2}+\frac{1}{2} A \mathbf{K} \cdot \mathbf{K}, \\
\stackrel{2}{\boldsymbol{\sigma}} & =\stackrel{4}{\mathbf{C}}: \stackrel{2}{\boldsymbol{\varepsilon}}^{e}, \quad R=R_{0}+H p, \quad a=H_{\phi} e+\beta \dot{e}, \quad \mathbf{b}=A \mathbf{K}+\kappa \dot{\mathbf{K}} .
\end{aligned}
$$

The viscoplastic potential is based on the yield function that introduces the equivalent stress measure $\sigma_{e q}$ and a threshold

$$
\Omega(\stackrel{2}{\boldsymbol{\sigma}, a-R})=\frac{K}{n+1}\left\langle\frac{\sigma_{e q}+a-R}{K}\right\rangle^{n+1}, \quad \stackrel{2}{\dot{\boldsymbol{\varepsilon}}^{p}}=\dot{p} \frac{\partial \sigma_{e q}}{\partial \boldsymbol{\sigma}}, \quad \dot{p}=\left\langle\frac{\sigma_{e q}+a-R}{K}\right\rangle^{n},
$$

where $\langle\cdot\rangle$ denotes the positive part of the quantity in brackets, and $K$ and $n$ are usual viscosity parameters. The decomposition (27) and the generalized balance (11) become

$$
a=H_{\phi}(\phi-p)+\beta(\dot{\phi}-\dot{p})=\boldsymbol{\nabla} \cdot(A \mathbf{K}+\kappa \dot{\mathbf{K}}) .
$$

We finally obtain the following linear partial differential equation, under the condition of plastic loading, in the absence of volume and inertial forces:

$$
H_{\phi} \phi-A \Delta \phi+\beta \dot{\phi}-\kappa \Delta \dot{\phi}=H_{\phi} p+\kappa \dot{p},
$$

where $\Delta$ is the Laplace operator. When the viscous parts are dropped in (27), the Helmholtz type equation used in strain gradient plasticity and damage [11, 14, 35] is retrieved. It is classically used for the regularization of strain localization phenomena. The rate dependent part in the previous equation is expected to be useful in the simulation of strain rate localization phenomena which occur for instance in strain aging materials [29].

Under plastic loading, the equivalent stress can then be decomposed into the following contributions:

$$
\sigma_{e q}=R-a+K \dot{p}^{1 / n}=R_{0}+H p-A \Delta \phi-\kappa \Delta \dot{\phi}+K \dot{p}^{1 / n} .
$$

If $\kappa=0$, the micromorphic model is retrieved. If, furthermore, the constraint $\phi \equiv p$ is enforced, Aifantis well-known strain gradient plasticity model is recovered. 


\subsection{Decomposition of the Generalized Strain Measures}

It is proposed now to consider the decomposition of the additional DOF and its gradient into elastic and plastic parts:

$$
\phi=\phi^{e}+\phi^{p}, \quad \mathbf{K}=\mathbf{K}^{e}+\mathbf{K}^{p} .
$$

The decomposition of $\phi$ itself is allowed only if it is an objective quantity. This would not apply for instance for $\phi \equiv \stackrel{2}{\mathbf{R}}$, the Cosserat microrotation. But it is allowed for a strain variable [16]. Such generalized kinematic decompositions were proposed in [16] for strain gradient, Cosserat and micromorphic media, also at finite deformation. It is generalized here for more general DOFs, possibly related to physically coupled phenomena.

The selected state variables then are

$$
\operatorname{STATE}=\left\{\tilde{\boldsymbol{\varepsilon}}^{e}, \quad \phi^{e}, \quad \mathbf{K}^{e}, \quad p\right\},
$$

which leads to the following Clausius-Duhem inequality:

$$
\begin{aligned}
\left(\stackrel{\boldsymbol{\sigma}}{2}-\rho \frac{\partial \psi}{\partial \boldsymbol{\varepsilon}^{e}}\right): \stackrel{2}{\dot{\boldsymbol{\varepsilon}}}^{e}+\left(a-\rho \frac{\partial \psi}{\partial \phi^{e}}\right) \dot{\phi}^{e}+(\mathbf{b}- & \left.\rho \frac{\partial \psi}{\partial \mathbf{K}^{e}}\right) \cdot \dot{\mathbf{K}}^{e} \\
& +\stackrel{2}{\boldsymbol{\sigma}}: \dot{\boldsymbol{\varepsilon}}^{p}+a \dot{\phi}^{p}+\mathbf{b} \cdot \dot{\mathbf{K}}^{p}-R \dot{p} \geq 0
\end{aligned}
$$

The retained state laws are

$$
\stackrel{2}{\boldsymbol{\sigma}}=\rho \frac{\partial \psi}{\partial \widetilde{\boldsymbol{\varepsilon}}^{e}}, \quad a=\rho \frac{\partial \psi}{\partial \phi^{e}}, \quad \mathbf{b}=\rho \frac{\partial \psi}{\partial \mathbf{K}^{e}} .
$$

The residual dissipation then is

$$
\stackrel{2}{\boldsymbol{\sigma}}: \stackrel{2}{\dot{\boldsymbol{\varepsilon}}}^{p}+a \dot{\phi}^{p}+\mathbf{b} \cdot \dot{\mathbf{K}}^{p}-R \dot{p} \geq 0 .
$$

A simple choice of dissipation potential is

$$
\Omega(\stackrel{2}{\boldsymbol{\sigma}}, R, a)=\frac{K}{n+1}\left\langle\frac{\sigma_{e q}+a-R}{K}\right\rangle^{n+1}+\frac{K_{a}}{m_{a}+1}\left(\frac{|a|}{K_{a}}\right)^{m_{a}+1}+\frac{K_{b}}{m_{b}+1}\left(\frac{b_{e q}}{K_{b}}\right)^{m_{b}+1},
$$

where $b_{e q}$ is a norm of $\mathbf{b}$ and from which the evolution rules are derived

$$
\begin{aligned}
& \stackrel{2}{\dot{\boldsymbol{\varepsilon}}}^{p}=\dot{p} \frac{\partial \sigma_{e q}}{\partial \stackrel{\boldsymbol{\sigma}}{2}}, \quad \dot{p}=-\frac{\partial \Omega}{\partial R}=\left\langle\frac{\sigma_{e q}+a-R}{K}\right\rangle^{n}, \\
& \dot{\phi}^{p}=\frac{\partial \Omega}{\partial a}=\dot{p}+\left(\frac{|a|}{K_{a}}\right)^{m_{a}} \operatorname{sign} a, \quad \dot{\mathbf{K}}^{p}=\frac{\partial \Omega}{\partial \mathbf{b}}=\left(\frac{b_{e q}}{K_{b}}\right)^{m_{b}} \frac{\partial b_{e q}}{\partial \mathbf{b}} .
\end{aligned}
$$


The time variation of the additional DOF therefore deviates from the cumulated plastic strain rate by a viscous term characterized by the material parameters $K_{a}$ and $m_{a}$. The residual dissipation rate becomes

$$
\left(\sigma_{e q}-R+a\right) \dot{p}+\frac{|a|^{m_{a}+1}}{K_{a}^{m_{a}}}+\left(\frac{b_{e q}}{K_{b}}\right)^{m_{b}} \frac{\partial b_{e q}}{\partial \mathbf{b}} \cdot \mathbf{b} \geq 0,
$$

which is indeed always positive.

Let us illustrate the type of partial differential equation provided by such a model. For that purpose, a simple quadratic free energy potential is chosen:

$$
\rho \psi=\frac{1}{2} \stackrel{2}{\varepsilon}^{e}: \stackrel{4}{\mathbf{C}}: \stackrel{2}{\varepsilon}^{e}+R_{0} p+\frac{1}{2} H p^{2}+\frac{1}{2} H_{\phi} \phi^{e 2}+\frac{1}{2} A \mathbf{K}^{e} \cdot \mathbf{K}^{e} .
$$

As a result, the corresponding state laws can be combined with the extra-balance equation (11):

$$
a=H_{\phi} \phi^{e}=\boldsymbol{\nabla} \cdot \mathbf{b}=\boldsymbol{\nabla} \cdot\left(A \mathbf{K}^{e}\right),
$$

which leads to the following partial differential equation, under the condition of material homogeneity:

$$
H_{\phi}\left(\phi-\phi^{p}\right)=A \Delta \phi-A \boldsymbol{\nabla} \cdot \mathbf{K}^{p}
$$

If $K_{a}=\infty$ (infinite viscosity), Eq. (42) shows that $\phi^{p}$ coincides with $p$. If, furthermore, $K_{b}=\infty$, the plastic part of $\mathbf{K}$ vanishes. The Eq. (45) then reduces to the Helmholtz-type equation (33) where $\beta$ and $\kappa$ are set to zero. An alternative expression of (45) can be worked out by taking the viscous laws into account

$$
a=K_{a}\left(\dot{\phi}^{p}-\dot{p}\right)=\boldsymbol{\nabla} \cdot \mathbf{b}=\boldsymbol{\nabla} \cdot K_{b} \dot{\mathbf{K}}^{p},
$$

which leads to the following partial differential equation:

$$
K_{a}\left(\dot{\phi}^{p}-\dot{p}\right)=K_{b} \boldsymbol{\nabla} \cdot \dot{\mathbf{K}}^{p} .
$$

When the elastic contributions $\phi^{e}$ and $\mathbf{K}^{e}$ are neglected, the previous equation reduces to

$$
K_{a}(\dot{\phi}-\dot{p})=K_{b} \Delta \dot{\phi},
$$

which is identical to (33) after taking $A=H_{\phi}=0$.

\section{Phase-Field Models for Elastoviscoplastic Materials}

In this section, the additional degree of freedom is a phase-field variable. We show how the constitutive framework for elastoviscoplastic materials can be embedded in the existing phase-field approach which combines diffusion and phase field equations to model the motion of boundaries between phases. The migration of interfaces 
and growth of precipitates are strongly influenced by the mechanical behavior of the phases.

One observes in current literature a strong endeavor to develop microstructure evolution simulation schemes coupled with complex mechanical material behavior ranging from heterogeneous elasticity to general elastoviscoplasticity. The main difficulty of such a task lies in the tight coupling between the complex interface evolutions and the fields, common to many moving boundary problems. The phase-field approach has emerged as a powerful method for easily tackling the morphological evolutions involved in phase transformations. Phase-field models have incorporated elasticity quite early [40] and have succeeded in predicting some complex microstructure evolutions driven by the interplay of diffusion and elasticity. It is only very recently that some phase-field models have been enriched with nonlinear mechanical behavior, extending the range of applications and materials which can be handled by the phase-field approach [3, 19, 20, 38].

There are essentially two ways of introducing linear and nonlinear mechanical constitutive equations into the standard phase-field approach:

1. The material behavior is described by a unified set of constitutive equations including material parameters that explicitly depend on the concentration or the phase variable. Each parameter is usually interpolated between the limit values known for each phase. This is the formulation adopted in the finite element simulations of Cahn-Hilliard like equations coupled with viscoplasticity in [38, 39] for tin-lead solders. The same methodology is used in [19, 20] to simulate the role of viscoplasticity on rafting of $\gamma^{\prime}$ precipitates in single crystal nickel base superalloys under load.

2. One distinct set of constitutive equations is attributed to each individual phase $k$ at any material point. Each phase at a material point then possesses its own stress/strain tensor $\underset{\boldsymbol{\sigma}}{\boldsymbol{\sigma}}, \underset{\boldsymbol{\varepsilon}_{k}}{\boldsymbol{\varepsilon}}$. The overall strain and stress quantities $\stackrel{2}{\boldsymbol{\sigma}}, \stackrel{2}{\boldsymbol{\varepsilon}}$ at this material point must then be averaged or interpolated from the values attributed to each phase. This is particularly important for points inside the smooth interface zone. At this stage, several mixture rules are available to perform this averaging or interpolation. This approach makes possible to mix different types of constitutive equations for each phase, like hyperelastic nonlinear behavior for one phase and conventional elastic-plastic model with internal variables for the other one. No correspondence of material parameters is needed between the phase behavior laws. This is the approach proposed in [37] for incorporating elasticity in a multiphase-field model. For that purpose, the authors resort to a well-known homogeneous stress hypothesis taken from homogenization theory in the mechanics of heterogeneous materials [8]. In the present work, we propose to generalize this procedure to nonlinear material behavior and to other mixture rules also taken from homogenization theory.

It must be emphasized that the latter procedure is very similar to what has already been proposed for handling diffusion in phase-field models by [26]. Two concentration fields $c_{\alpha}$ and $c_{\beta}$ are indeed introduced, and the real concentration field is obtained by a mixture rule together with an internal constraint on the diffusion 
potentials. Introducing two concentration fields gives an additional degree of freedom for controlling the energy of the interface with respect to its thickness. If this possibility is not obvious when mechanics is introduced, adding a degree of freedom for describing the stresses/strains within a diffuse interface could be valuable to get rid of some spurious effects due to unrealistic interface thickness.

\subsection{Coupling with Diffusion}

In the context of mass diffusion and phase-field evolution, the local form of the energy principle is

$$
\dot{e}=\stackrel{2}{\boldsymbol{\sigma}}: \stackrel{2}{\dot{\varepsilon}}+a \dot{\phi}+\mathbf{b} \cdot \nabla \dot{\phi}
$$

The total strain is partitioned into the elastic strain $\stackrel{2}{\varepsilon}^{e}$, the eigenstrain $\stackrel{2}{\varepsilon}^{\star}$ due to phase transformation and the plastic strain $\stackrel{2}{\varepsilon}^{p}$ :

$$
\stackrel{2}{\boldsymbol{\varepsilon}}=\stackrel{2}{\boldsymbol{\varepsilon}}^{e}+\stackrel{2}{\boldsymbol{\varepsilon}}^{\star}+\stackrel{2}{\boldsymbol{\varepsilon}}^{p}
$$

According to the thermodynamics of irreversible processes, the second law states that the variation of entropy is always larger than or equal to the rate of entropy flux induced by diffusion:

$$
T \dot{\eta}-\boldsymbol{\nabla} \cdot(\mu \mathbf{J}) \geqslant 0,
$$

where $\mathbf{J}$ is the diffusion flux and $\mu$ is the chemical potential. The conservation law for mass diffusion is then

$$
\dot{c}=-\nabla \cdot \mathbf{J} .
$$

Accordingly, the fundamental inequality containing first and second principles in the isothermal case is written as

$$
-\rho \dot{\psi}+\stackrel{2}{\boldsymbol{\sigma}}: \stackrel{2}{\dot{\boldsymbol{\varepsilon}}}+a \dot{\phi}+\mathbf{b} \cdot \boldsymbol{\nabla} \dot{\phi}+\mu \dot{c}-\mathbf{J} \cdot \nabla \mu \geqslant 0 .
$$

Assuming that the free energy density depends on the order parameter $\phi$ and its gradient, the concentration $c$, the elastic strain $\stackrel{2}{\boldsymbol{\varepsilon}}^{e}$ and the set of internal variables $V_{k}$ associated to material hardening ${ }^{1}$ :

$$
S T A T E=\left\{\phi, \quad \nabla \phi, \quad c, \quad \stackrel{2}{\varepsilon}^{e}, \quad V_{k}\right\} .
$$

The Clausius-Duhem inequality now becomes:

$$
\begin{aligned}
\left(a-\rho \frac{\partial \psi}{\partial \phi}\right) \dot{\phi}+\left(\mathbf{b}-\rho \frac{\partial \psi}{\partial \boldsymbol{\nabla} \phi}\right) & \cdot \boldsymbol{\nabla} \dot{\phi}+\left(\mu-\rho \frac{\partial \psi}{\partial c}\right) \dot{c} \\
& +\left(\stackrel{2}{\boldsymbol{\sigma}}-\frac{\partial \psi}{\partial \boldsymbol{\varepsilon}^{e}}\right): \dot{\boldsymbol{\varepsilon}}^{e}-\mathbf{J} . \boldsymbol{\nabla} \mu+\stackrel{2}{\boldsymbol{\sigma}}: \dot{\boldsymbol{\varepsilon}}^{p}-\rho \frac{\partial \psi}{\partial V_{k}} \dot{V}_{k} \geqslant 0
\end{aligned}
$$

\footnotetext{
${ }^{1}$ In this section, the notation for internal variables is changed to $\left(V_{k}\right)_{k \in\{\alpha, \beta\}}$ since $\alpha$ is now an index denoting one phase.
} 
The following reversible mechanisms and corresponding state laws are chosen:

$$
\mathbf{b}=\rho \frac{\partial \psi}{\partial \boldsymbol{\nabla} \phi}, \quad \mu=\rho \frac{\partial \psi}{\partial c}, \quad \stackrel{2}{\boldsymbol{\sigma}}=\rho \frac{\partial \psi}{\partial \boldsymbol{\varepsilon}^{e}}, \quad A_{k}:=\rho \frac{\partial \psi}{\partial V_{k}}
$$

The residual dissipation then is

$$
\left(a-\rho \frac{\partial \psi}{\partial \phi}\right) \dot{\phi}-\mathbf{J} \cdot \nabla \mu+\stackrel{2}{\sigma}: \dot{\boldsymbol{\varepsilon}}^{p}-A_{k} \dot{V}_{k} \geqslant 0 .
$$

Three contributions appear in the above residual dissipation rate. The first is the phase-field dissipation, associated with configuration changes of atoms and related to the evolution of the order parameter:

$$
D_{\phi}=a^{v} \dot{\phi} \quad \text { with } \quad a^{v}=a-\rho \frac{\partial \psi}{\partial \phi}
$$

where $a^{v}$ is the chemical force associated with the dissipative processes [22]. The second contribution is the chemical dissipation due to diffusion, associated with mass transport. The last contribution is the mechanical dissipation, as discussed earlier.

An efficient way of defining the complementary laws related to the dissipative processes and ensuring the positivity of the dissipation for any thermodynamic process is to assume the existence of a dissipation potential $\Omega\left(a^{v}, \nabla \mu \stackrel{2}{\sigma}, A_{k}\right)$, which is a convex function of its arguments:

$$
\dot{\phi}=\frac{\partial \Omega}{\partial a^{v}}, \quad \mathbf{J}=-\frac{\partial \Omega}{\partial \boldsymbol{\nabla} \mu}, \quad \dot{V}_{k}=-\frac{\partial \Omega}{\partial A_{k}}, \quad \stackrel{2}{\dot{\boldsymbol{\varepsilon}}^{p}}=\frac{\partial \Omega}{\partial \stackrel{2}{\boldsymbol{\sigma}}} .
$$

These equations represent the evolution law for the order parameter, the diffusion flux as well as the evolution laws for the internal variables.

\subsection{Partition of Free Energy and Dissipation Potential}

The total free energy is postulated to have the form of a Ginzburg-Landau free energy functional accounting for interfaces through the square of the order parameter gradient. The free energy density $\psi$ is then split into a chemical free energy density $\psi_{\mathrm{ch}}$, a coherent mechanical energy density $\psi_{\text {mech }}$, and the square of the order parameter gradient:

$$
\rho \psi\left(\phi, \nabla \phi, c, \stackrel{2}{\varepsilon}^{e}, V_{k}\right)=\rho \psi_{\mathrm{ch}}(\phi, c)+\rho \psi_{\mathrm{mech}}\left(\phi, c, \stackrel{2}{\boldsymbol{\varepsilon}}, V_{k}\right)+\frac{A}{2} \boldsymbol{\nabla} \phi \cdot \boldsymbol{\nabla} \phi .
$$

The irreversible part of the behavior is described by the dissipation potential, which can be split into three parts related to the three contributions in the residual 
dissipation in Eq.57): the phase-field part $\Omega_{\phi}\left(\phi, c, a^{v}\right)$, the chemical part $\Omega_{c}(\phi, c, \nabla \mu)$ and the mechanical dissipation potential $\Omega_{\text {mech }}\left(\phi, c, \stackrel{2}{\sigma}, A_{k}\right)$ :

$$
\Omega\left(a^{v}, \nabla \mu, \phi, c, \stackrel{2}{\sigma}, A_{k}\right)=\Omega_{\phi}\left(c, \phi, a^{v}\right)+\Omega_{c}(c, \phi, \nabla \mu)+\Omega_{\operatorname{mech}}\left(\phi, c, \stackrel{2}{\sigma}, A_{k}\right) .
$$

The chemical free energy density $\psi_{\mathrm{ch}}$ of a binary alloy is a function of the order parameter $\phi$ and of the concentration field $c$. The coexistence of both phases $\alpha$ and $\beta$ discriminated by $\phi$ is possible if $\psi_{\mathrm{ch}}$ is non-convex with respect to $\phi$. Following [25], $\psi_{\mathrm{ch}}$ is built with the free energy densities of the two phases $\psi_{\alpha}$ and $\psi_{\beta}$ as follows:

$$
\psi_{\mathrm{ch}}(\phi, c)=h(\phi) \psi_{\alpha}(c)+(1-h(\phi)) \psi_{\beta}(c)+W g(\phi) .
$$

Here, the interpolating function $h(\phi)$ is chosen as $h(\phi)=\phi^{2}(3-2 \phi)$, and $g(\phi)=$ $\phi^{2}(1-\phi)^{2}$ is the double well potential accounting for the free energy penalty of the interface. The height $W$ of the potential barrier is related to the interfacial energy $\sigma$ and the interfacial thickness $\delta$ as $W=6 \Lambda \sigma / \delta$. Assuming that the interface region ranges from $\theta$ to $1-\theta$, then $\Lambda=\log ((1-\theta) / \theta)$. In the present work $\theta=0.05$ [2, 25].

The densities $\psi_{\alpha}$ and $\psi_{\beta}$ are chosen to be quadratic functions of the concentration only:

$$
\rho \psi_{\alpha}(c)=\frac{k_{\alpha}}{2}\left(c-a_{\alpha}\right)^{2} \quad \text { and } \quad \rho \psi_{\beta}(c)=\frac{k_{\beta}}{2}\left(c-a_{\beta}\right)^{2},
$$

where $a_{\alpha}$ and $a_{\beta}$ are the unstressed equilibrium concentrations of both phases which correspond respectively to the minima of $\psi_{\alpha}$ and $\psi_{\beta}$ in the present model. $k_{\alpha}$ and $k_{\beta}$ are the curvatures of the free energies.

Quadratic expressions are chosen for the chemical dissipation, which ensures the positivity of the dissipation rate:

$$
\Omega_{\phi}\left(a^{v}\right)=\frac{1}{2}(1 / \beta) a^{v 2} \quad \text { and } \quad \Omega_{c}(\nabla \mu)=\frac{1}{2} L(\phi) \nabla \mu \cdot \nabla \mu
$$

where $a^{v}$ is given by Eq. (58), $\beta$ is inversely proportional to the interface mobility and $L(\phi)$ is the Onsager coefficient, related to the chemical diffusivities $D_{\alpha}$ and $D_{\beta}$ in both phases by means of the interpolation function $h(\phi)$ as

$$
L(\phi)=h(\phi) D_{\alpha} / k_{\alpha}+(1-h(\phi)) D_{\beta} / k_{\beta} .
$$

The state laws and evolution equations for the phase-field and chemical contributions can be derived as

$$
\begin{gathered}
\mathbf{b}=A \boldsymbol{\nabla} \phi, \quad \mu=\rho \frac{\partial \psi_{\mathrm{ch}}}{\partial c}+\rho \frac{\partial \psi_{\mathrm{mech}}}{\partial c} \\
\dot{\phi}=\frac{1}{\beta} a^{v}=\frac{1}{\beta}\left(a-\rho \frac{\partial \psi_{\mathrm{ch}}}{\partial \phi}-\rho \frac{\partial \psi_{\mathrm{mech}}}{\partial \phi}\right), \quad \mathbf{J}=-L(\phi) \nabla \mu .
\end{gathered}
$$


Substituting the previous equations into the balance equations for generalized stresses and mass concentration, the Ginzburg-Landau and usual diffusion equations are retrieved, which represent respectively the evolution equations for order parameter and concentration:

$$
\begin{gathered}
\boldsymbol{\nabla} \cdot \mathbf{b}-a=-\beta \dot{\phi}+\boldsymbol{\nabla} \cdot(A \boldsymbol{\nabla} \phi)-\rho \frac{\partial \psi_{\mathrm{ch}}}{\partial \phi}-\rho \frac{\partial \psi_{\mathrm{mech}}}{\partial \phi}=0, \\
\dot{c}=-\boldsymbol{\nabla} \cdot(-L(\phi) \boldsymbol{\nabla} \mu)=-\boldsymbol{\nabla} \cdot\left(-L(\phi)\left(\boldsymbol{\nabla} \frac{\partial \rho \psi_{\mathrm{ch}}}{\partial c}+\nabla \frac{\partial \rho \psi_{\mathrm{mech}}}{\partial c}\right)\right) .
\end{gathered}
$$

Note the coupling of mechanics and diffusion and phase-field evolution through the partial derivatives of the mechanical free energy with respect to concentration and order parameter.

\subsection{Multi-phase Approach for the Mechanical Contribution}

The second contribution to the free energy density is due to mechanical effects. Assuming that elastic behavior and hardening are uncoupled, the mechanical part of the free energy density $\rho \psi_{\text {mech }}$ is decomposed into a coherent elastic energy density $\rho \psi_{e}$ and a plastic part $\rho \psi_{p}$ as

$$
\rho \psi_{\mathrm{mech}}\left(\phi, c, \stackrel{2}{\boldsymbol{\varepsilon}}, V_{k}\right)=\rho \psi_{e}(\phi, c, \stackrel{2}{\boldsymbol{\varepsilon}})+\rho \psi_{p}\left(\phi, c, V_{k}\right) .
$$

Moreover, the irreversible mechanical behavior, related to the dissipative processes, is obtained by a plastic dissipation potential $\Omega_{\text {mech }}\left(\phi, c, \stackrel{2}{\sigma}, A_{k}\right)$. It is assumed to be a function of order parameter, concentration, Cauchy stress tensor as well as the set of thermodynamic force associated variables $A_{k}$ in order to describe the hardening state in each phase.

In the diffuse interface region where both phases coexist, we propose to use wellknown results of homogenization theory to interpolate the local behavior. The homogenization procedure in the mechanics of heterogeneous materials consists in replacing an heterogeneous medium by an equivalent homogeneous one, which is defined by an effective constitutive law relating the macroscopic variables, namely macroscopic stress $\stackrel{2}{\sigma}$ and strain $\stackrel{2}{\varepsilon}$ tensors, which are obtained by averaging the corresponding non-uniform local stress and strain in each phase. Each material point within a diffuse interface can be seen as a local mixture of the two abutting phases $\alpha$ and $\beta$ with proportions given by complementary functions of $\phi$. The strain and stress at each material point are then defined by the following mixture laws which would proceed from space averaging in a conventional homogenization problem, but which must be seen as arbitrary interpolations in the present case:

$$
\stackrel{2}{\boldsymbol{\varepsilon}}=\chi \stackrel{2}{\boldsymbol{\varepsilon}}_{\alpha}+(1-\chi)^{2} \stackrel{\boldsymbol{\varepsilon}}{\beta}_{\beta} \quad \text { and } \quad \stackrel{2}{\boldsymbol{\sigma}}=\chi \stackrel{2}{\boldsymbol{\sigma}}_{\alpha}+(1-\chi)^{\boldsymbol{2}} \stackrel{2}{\beta}_{1}
$$


where $\stackrel{2}{\varepsilon}_{\alpha}, \stackrel{2}{\varepsilon}_{\beta}$ are local fictitious strains and $\stackrel{2}{\sigma}_{\alpha}, \stackrel{2}{\sigma}_{\beta}$ are local fictitious stresses in $\alpha$ and $\beta$ phases respectively and $\chi(\mathbf{x}, t)$ is a shape function which must take the value 0 in the $\beta$-phase and 1 in the $\alpha$-phase. The following choice is made in the phase field context:

$$
\chi(\mathbf{x}, t) \equiv \phi(\mathbf{x}, t) .
$$

The partition hypothesis, already used for the effective total strain tensor in Eq. (51), requires, in a similar way, a decomposition of the total strain in each phase into elastic, transformation and plastic parts:

$$
\stackrel{2}{\boldsymbol{\varepsilon}_{\alpha}}=\stackrel{2}{\boldsymbol{\varepsilon}_{\alpha}^{e}}+\stackrel{2}{\boldsymbol{\varepsilon}_{\alpha}^{\star}}+\stackrel{2}{\boldsymbol{\varepsilon}_{\alpha}} \text { and } \quad \stackrel{2}{\boldsymbol{\varepsilon}_{\beta}}=\stackrel{2}{\boldsymbol{\varepsilon}_{\beta}^{e}}+\stackrel{2}{\boldsymbol{\varepsilon}_{\beta}^{\star}}+\stackrel{2}{\boldsymbol{\varepsilon}_{\beta}^{p}},
$$

where each point may depend on the local concentration $c$, but not on order parameter $\phi$. In the proposed model, the elastoplastic and phase-field behaviors of each phase are treated independently and the effective behavior is obtained using homogenization relation (71). It is assumed that the mechanical state of $\alpha$ and $\beta$ phases at a given time are completely described by a finite number of local state variables $\left(\varepsilon_{k}^{e}, V_{k}\right)$ defined at each material point. The set of internal variables $V_{k}$, of scalar or tensorial nature, represents the state of hardening of phase $k$ : for instance, a scalar isotropic hardening variable, and a tensorial kinematic hardening variable. According to the homogenization theory, the effective elastic and plastic free energy densities are given by the rule of mixtures as follows:

$$
\begin{gathered}
\rho \psi_{e}\left(\phi, c,{ }^{2}\right)=\phi \rho \psi_{e \alpha}\left(c, 2^{e}\right)+(1-\phi) \rho \psi_{e \beta}\left(c,{ }^{e} \boldsymbol{\varepsilon}_{\beta}^{e}\right), \\
\rho \psi_{p}\left(\phi, c, V_{k}\right)=\phi \rho \psi_{p \alpha}\left(c, V_{\alpha}\right)+(1-\phi) \rho \psi_{p \beta}\left(c, V_{\beta}\right) .
\end{gathered}
$$

Similarly, a mixture rule is used to mix the dissipation potentials of the individual phases:

$$
\Omega_{\mathrm{mech}}\left(\phi, c, \stackrel{2}{\boldsymbol{\sigma}}, A_{k}\right)=\phi \Omega_{\mathrm{mech} \alpha}\left(c, \stackrel{2}{\boldsymbol{\sigma}_{\alpha}}, A_{\alpha}\right)+(1-\phi) \Omega_{\mathrm{mech} \beta}\left(c, \stackrel{2}{\sigma}_{\beta}, A_{\beta}\right),
$$

where the $A_{\alpha, \beta}$ are the thermodynamic forces associated with the internal variables attributed to each phase.

Knowing the free energy and dissipation potentials, the evolution of all variables can be computed. The remaining questions is the way of estimating the previously defined fictitious stress and strain tensors $\stackrel{2}{\varepsilon}_{\alpha, \beta}, \stackrel{2}{\sigma}_{\alpha, \beta}$ from the knowledge of the stress and strain tensors $\stackrel{2}{\varepsilon}$ and $\stackrel{2}{\sigma}$. Several homogenization schemes exist in the literature that can be used to define these new fictitious variables. The most simple schemes are the Voigt/Taylor and Reuss/Static models. We develop the Voigt/Taylor scenario in the sequel. 


\subsection{Voigt/Taylor Model Coupled Phase-Field Mechanical Theory}

According to Voigt's scheme, the fictitious strains are not distinguished from the local strain. The local stress is then computed in terms of the fictitious stress tensors by averaging with respect to both phases weighted by the volume fractions:

$$
\stackrel{2}{\boldsymbol{\sigma}}=\phi \stackrel{2}{\boldsymbol{\sigma}}_{\alpha}+(1-\phi) \stackrel{2}{\boldsymbol{\sigma}_{\beta}}, \quad \stackrel{2}{\boldsymbol{\varepsilon}}=\stackrel{2}{\boldsymbol{\varepsilon}}_{\alpha}=\stackrel{2}{\boldsymbol{\varepsilon}}_{\beta}
$$

The stresses of both phases $\stackrel{2}{\boldsymbol{\sigma}_{\alpha}}$ and $\stackrel{2}{\sigma}_{\beta}$ are given by Hooke's law for each phase:

$$
\stackrel{2}{\stackrel{\boldsymbol{\sigma}}{\alpha}_{\alpha}}=\stackrel{4}{\mathbf{C}}_{\alpha}:\left(\stackrel{2}{\boldsymbol{\varepsilon}}_{\alpha}-\stackrel{2}{\boldsymbol{\varepsilon}}_{\alpha}^{\star}-\stackrel{2}{\boldsymbol{\varepsilon}}_{\alpha}^{p}\right), \quad \stackrel{2}{\boldsymbol{\sigma}}_{\beta}=\stackrel{4}{\mathbf{C}}_{\beta}:\left(\stackrel{2}{\boldsymbol{\varepsilon}}_{\beta}-\stackrel{2}{\boldsymbol{\varepsilon}}_{\beta}^{\star}-\stackrel{2}{\boldsymbol{\varepsilon}}_{\beta}^{p}\right),
$$

where $\stackrel{4}{\mathbf{C}_{\alpha}}$ and $\stackrel{4}{\mathbf{C}}_{\beta}$ are respectively the tensor of elasticity moduli in $\alpha$ and $\beta$ phases. As a result,

$$
\stackrel{2}{\boldsymbol{\sigma}}=\phi \stackrel{4}{\mathbf{C}}_{\alpha}:\left(\stackrel{2}{\boldsymbol{\varepsilon}}_{\alpha}-\stackrel{2}{\boldsymbol{\varepsilon}}_{\alpha}^{\star}-\stackrel{2}{\boldsymbol{\varepsilon}}_{\alpha}^{p}\right)+(1-\phi) \stackrel{4}{\mathbf{C}}{ }_{\beta}:\left(\stackrel{2}{\boldsymbol{\varepsilon}}_{\beta}-\stackrel{2}{\boldsymbol{\varepsilon}}_{\beta}^{\star}-\stackrel{2}{\boldsymbol{\varepsilon}}_{\beta}^{p}\right)
$$

From the above relation, it follows that the strain-stress relationship in the homogeneous effective medium obeys Hooke's law with the following equation:

$$
\stackrel{2}{\boldsymbol{\sigma}}=\stackrel{4}{\mathbf{C}}_{\mathrm{eff}}:\left(\stackrel{2}{\boldsymbol{\varepsilon}}-\stackrel{2}{\boldsymbol{\varepsilon}}^{p}-\stackrel{2}{\boldsymbol{\varepsilon}}^{\star}\right),
$$

where the effective elasticity tensor $\stackrel{4}{\mathbf{C}}_{\text {eff }}$ is obtained from the mixture rule of the elasticity matrix for both phases:

$$
\stackrel{4}{\mathbf{C}}_{\mathrm{eff}}=\phi \stackrel{4}{\mathbf{C}}_{\alpha}+(1-\phi) \stackrel{4}{\mathbf{C}}_{\beta}
$$

and the effective eigenstrain $\stackrel{2}{\varepsilon}^{\star}$ and plastic strain $\stackrel{2}{\varepsilon}^{p}$ vary continuously between their respective values in the bulk phases as follows:

$$
\begin{aligned}
& \stackrel{2}{\boldsymbol{\varepsilon}}^{\star}=\stackrel{4}{\mathbf{C}_{\mathrm{eff}}^{-1}}:\left(\phi \stackrel{4}{\mathbf{C}}{ }_{\alpha}: \stackrel{2}{\boldsymbol{\varepsilon}}_{\alpha}^{\star}+(1-\phi) \stackrel{4}{\mathbf{C}}_{\beta}: \stackrel{2}{\boldsymbol{\varepsilon}}_{\beta}^{\star}\right), \\
& \stackrel{4}{\boldsymbol{\varepsilon}^{p}}=\stackrel{4}{\mathbf{C}}_{\mathrm{eff}}^{-1}:\left(\phi \stackrel{4}{\mathbf{C}}_{\alpha}: \stackrel{2}{\boldsymbol{\varepsilon}}_{\alpha}^{p}+(1-\phi) \stackrel{4}{\mathbf{C}}_{\beta}: \stackrel{2}{\boldsymbol{\varepsilon}}_{\beta}^{p}\right)
\end{aligned}
$$

In the case of non-homogeneous elasticity, it must be noted that $\stackrel{2}{\star}^{\star}$ and $\stackrel{2}{\boldsymbol{\varepsilon}}^{p}$ are not the average of their respective values for each phase.

The proposed approach differs from the one most commonly used in phase-field models, as popularized by Khachaturyan and co-workers, e. g. [24]. The latter rely on mixture laws for all quantities within the interface, including the elastic moduli, the transformation and plastic strain. The effect of these different choices on the simulation of moving phase boundaries has been tested in [3] and [4]. In particular, the impact of plasticity on the kinetics of precipitate growth has been evidenced. 


\section{Conclusion}

The general thermomechanical setting for modeling size effects in the mechanics and thermodynamics of materials is based on the main assumption that microstructure effects can be accounted for by the introduction of additional degrees of freedom in addition to displacement, temperature and concentration. The additional DOF and its gradient are expected to contribute to the power of internal forces of the medium and to arise in the energy local balance equations and/or entropy inequality. They induce generalized stresses that fulfill an additional balance equation with associated extra boundary conditions. A clear separation between balance equations and constitutive functionals is adopted in the formulation. Constitutive equations derive from the definition of a specific free energy density and dissipation potential.

The crossing of mechanical and physical approaches turns out to be fertile in providing motivated coupling between both kinds of phenomena. As an example, we have shown that the mechanics of heterogeneous materials can be useful to develop a sophisticated and flexible constitutive framework of coupled viscoplasticity and diffusion.

It was not possible to address applications that already exist in this context. In particular, the presented models predict that viscoplasticity affects the morphology and kinetics of precipitate growth in metals or during oxidation [2, 4, 20].

Special attention must now be dedicated to more precise description of coherent vs. incoherent interfaces [5, 23, 34], and the associated specific interface conditions that can be deduced from asymptotic analysis of phase-field models. On the other hand, the targeted applications of strain gradient plasticity are crystal plasticity and grain boundary migration $[1,9,28]$, whereas strain rate gradients are thought to be relevant for aging materials [29].

\section{References}

[1] Abrivard, G.: A coupled crystal plasticity-phase field formulation to describe microstructural evolution in polycrystalline aggregates. PhD, Mines ParisTech (2009)

[2] Ammar, K., Appolaire, B., Cailletaud, G., Feyel, F., Forest, F.: Finite element formulation of a phase field model based on the concept of generalized stresses. Computational Materials Science 45, 800-805 (2009)

[3] Ammar, K., Appolaire, B., Cailletaud, G., Forest, S.: Combining phase field approach and homogenization methods for modelling phase transformation in elastoplastic media. European Journal of Computational Mechanics 18, 485-523 (2009)

[4] Ammar, K., Appolaire, B., Cailletaud, G., Forest, S.: Phase field modeling of elastoplastic deformation induced by diffusion controlled growth of a misfitting spherical precipitate. Philosophical Magazine Letters (2011)

[5] Appolaire, B., Aeby-Gautier, E., Teixeira, J.D., Dehmas, M., Denis, S.: Non-coherent interfaces in diffuse interface models. Philosophical Magazine 90, 461-483 (2010)

[6] Aslan, O., Forest, S.: Crack growth modelling in single crystals based on higher order continua. Computational Materials Science 45, 756-761 (2009) 
[7] Aslan, O., Forest, S.: The micromorphic versus phase field approach to gradient plasticity and damage with application to cracking in metal single crystals. In: de Borst, R., Ramm, E. (eds.) Multiscale Methods in Computational Mechanics. LNACM, vol. 55, pp. 135-154. Springer, Heidelberg (2011)

[8] Besson, J., Cailletaud, G., Chaboche, J.L., Forest, S., Blétry, M.: Non-Linear Mechanics of Materials. Series: Solid Mechanics and Its Applications, vol. 167, p. 433. Springer, Heidelberg (2009)

[9] Cordero, N., Gaubert, A., Forest, S., Busso, E., Gallerneau, F., Kruch, S.: Size effects in generalised continuum crystal plasticity for two-phase laminates. Journal of the Mechanics and Physics of Solids 58, 1963-1994 (2010)

[10] Ehlers, W., Volk, W.: On theoretical and numerical methods in the theory of porous media based on polar and non-polar elasto-plastic solid materials. International Journal of Solids and Structures 35, 4597-4617 (1998)

[11] Engelen, R., Geers, M., Baaijens, F.: Nonlocal implicit gradient-enhanced elastoplasticity for the modelling of softening behaviour. International Journal of Plasticity $19,403-433$ (2003)

[12] Eringen, A., Suhubi, E.: Nonlinear theory of simple microelastic solids. International Journal of Engineering Science 203, 189-203, 389-404 (1964)

[13] Finel, A., Le Bouar, Y., Gaubert, A., Salman, U.: Phase field methods: Microstructures, mechanical properties and complexity. Comptes Rendus Physique 11, 245-256 (2010)

[14] Forest, S.: The micromorphic approach for gradient elasticity, viscoplasticity and damage. ASCE Journal of Engineering Mechanics 135, 117-131 (2009)

[15] Forest, S., Aifantis, E.C.: Some links between recent gradient thermo-elasto-plasticity theories and the thermomechanics of generalized continua. International Journal of Solids and Structures 47, 3367-3376 (2010)

[16] Forest, S., Sievert, R.: Nonlinear microstrain theories. International Journal of Solids and Structures 43, 7224-7245 (2006)

[17] Frémond, M., Nedjar, B.: Damage, gradient of damage and principle of virtual power. International Journal of Solids and Structures 33, 1083-1103 (1996)

[18] Fried, E., Gurtin, M.: Continuum theory of thermally induced phase transitions based on an order parameter. Physica D 68, 326-343 (1993)

[19] Gaubert, A., Finel, A., Le Bouar, Y., Boussinot, G.: Viscoplastic phase field modellling of rafting in ni base superalloys. In: Continuum Models and Discrete Systems CMDS11, pp. 161-166. Mines Paris Les Presses (2008)

[20] Gaubert, A., Le Bouar, Y., Finel, A.: Coupling phase field and viscoplasticity to study rafting in ni-based superalloys. Philosophical Magazine 90, 375-404 (2010)

[21] Germain, P.: La méthode des puissances virtuelles en mécanique des milieux continus, première partie : théorie du second gradient. Journal de Mécanique 12, 235-274 (1973)

[22] Gurtin, M.: Generalized Ginzburg-Landau and Cahn-Hilliard equations based on a microforce balance. Physica D 92, 178-192 (1996)

[23] Johnson, W.C., Alexander, J.I.D.: Interfacial conditions for thermomechanical equilibrium in two-phase crystals. Journal of Applied Physics 9, 2735-2746 (1986)

[24] Khachaturyan, A.: Theory of Structural Transformations in Solids. John Wiley \& Sons, New York (1983)

[25] Kim, S., Kim, W., Suzuki, T.: Interfacial compositions of solid and liquid in a phasefield model with finite interface thickness for isothermal solidification in binary alloys. Physical Review E 58(3), 3316-3323 (1998)

[26] Kim, S., Kim, W., Suzuki, T.: Phase-field model for binary alloys. Physical Review E 60(6), 7186-7197 (1999) 
[27] Maugin, G.: The method of virtual power in continuum mechanics: Application to coupled fields. Acta Mechanica 35, 1-70 (1980)

[28] Mayeur, J., McDowell, D., Bammann, D.: Dislocation-based micropolar single crystal plasticity: Comparison of multi- and single criterion theories. Journal of the Mechanics and Physics of Solids 59, 398-422 (2011)

[29] Mazière, M., Besson, J., Forest, S., Tanguy, B., Chalons, H., Vogel, F.: Numerical aspects in the finite element simulation of the portevin-le chatelier effect. Computer Methods in Applied Mechanics and Engineering 199, 734-754 (2010)

[30] Miehe, C.: A multi-field incremental variational framework for gradient-extended standard dissipative solids. Journal of the Mechanics and Physics of Solids 59, 898-923 (2011)

[31] Miehe, C., Welchinger, F., Hofacker, M.: A phase field model of electromechanical fracture. Journal of the Mechanics and Physics of Solids 58, 1716-1740 (2010)

[32] Miehe, C., Welchinger, F., Hofacker, M.: Thermodynamically-consistent phase field models of fracture: Variational principles and multifield FE implementations. International Journal for Numerical Methods in Engineering 83, 1273-1311 (2010)

[33] Mindlin, R.: Micro-structure in linear elasticity. Archive for Rational Mechanics and Analysis 16, 51-78 (1964)

[34] Murdoch, A.I.: A thermodynamical theory of elastic material interfaces. The Quarterly Journal of Mechanics and Applied Mathematics 29, 245-275 (1978)

[35] Peerlings, R., Geers, M.: Borst, R., Brekelmans, W. critical comparison of nonlocal and gradient-enhanced softening continua. International Journal of Solids and Structures 38, 7723-7746 (2001)

[36] Rajagopal, A., Fischer, P., Kuhl, E., Steinmann, P.: Natural element analysis of the Cahn-Hilliard phase-field model. Computational Mechanics 46, 471-493 (2010)

[37] Steinbach, I., Apel, M.: Multi phase field model for solid state transformation with elastic strain. Physica D 217, 153-160 (2006)

[38] Ubachs, R., Schreurs, P., Geers, M.: A nonlocal diffuse interface model for microstructure evolution of tin-lead solder. Journal of the Mechanics and Physics of Solids 52, 1763-1792 (2004)

[39] Ubachs, R., Schreurs, P., Geers, M.: Elasto-viscoplastic nonlocal damage modelling of thermal fatigue in anisotropic lead-free solder. Mechanics of Materials 39, 685-701 (2007)

[40] Wang, Y., Chen, L.Q., Khachaturyan, A.: Kinetics of strain-induced morphological transformation in cubic alloys with a miscibility gap. Acta Metallurgica et Materialia 41, 279-296 (1993) 